INPLASY

PROTOCOL

To cite: Qin et al. Effects of Spore Powder of Ganoderma Lucidum on CaSR and apoptosis-related proteins in hippocampus tissue of epilepsy following dementia: a protocol of systematic review. Inplasy protocol 202070041. doi:

10.37766/

inplasy2020.7.0041

Received: 11 July 2020

Published: 11 July 2020

Corresponding author:

Shu-ping Zhang

heweizong36@21cn.com

Author Affiliation:

First Affiliated Hospital of Jiamusi University

Support: HLJNSF (C201242).

Review Stage at time of this submission: The review has not yet started.

Conflicts of interest: None.

\section{Effects of Spore Powder of Ganoderma Lucidum on CaSR and apoptosis-related proteins in hippocampus tissue of epilepsy following dementia: a protocol of systematic review}

Qin, LH1'; Wang, C2; Jiang, XX33 Song, Y4; Feng, Y5; Qin, LW6; Zhang, SP7.

Review question / Objective: Does Spore Powder of Ganoderma Lucidum (SPGL) affect on CaSR and apoptosisrelated proteins (ARP) in hippocampus tissue of epilepsy following dementia?

Condition being studied: Spore Powder of Ganoderma Lucidum; CaSR; apoptosis-related proteins.

Information sources: Electronic bibliographic databases - We will search animal studies of question for SPGL on CaSR and ARP in hippocampus tissue of rats with epilepsy following dementia in Cochrane Library, EMBASE, MEDLINE, CINAHL, AMED, and CNKI from inception to the present. We present search strategy with details for Cochrane Library. We will adapt similar search strategy to other electronic bibliographic databases. Other sources - We will also search other sources to avoid missing potential studies, such as conference proceedings, associated references lists of included studies, and ongoing trials from websites of clinical trial registry.

INPLASY registration number: This protocol was registered with the International Platform of Registered Systematic Review and Meta-Analysis Protocols (INPLASY) on 11 July 2020 and was last updated on 11 July 2020 (registration number INPLASY202070041).

\section{INTRODUCTION}

Review question / Objective: Does Spore Powder of Ganoderma Lucidum (SPGL) affect on CaSR and apoptosis-related proteins (ARP) in hippocampus tissue of epilepsy following dementia?

Condition being studied: Spore Powder of Ganoderma Lucidum; CaSR; apoptosisrelated proteins. 


\section{METHODS}

Participant or population: Rats confirmed with epilepsy after dementia will be included in this study.

Intervention: In the experimental group, any forms of SPGL were used as the only treatment.

Comparator: In the control group, no restrictions were applied to the comparator, except SPGL.

Study designs to be included: All eligible case-control studies (CCSs) on assessing the effects of SPGL on CaSR and ARP in hippocampus tissue of rats with epilepsy and dementia.

Eligibility criteria: All eligible CCSs on assessing the effects of SPGL on CaSR and ARP in hippocampus tissue of rats with epilepsy following dementia will be considered for inclusion.

Information sources: Electronic bibliographic databases - We will search animal studies of question for SPGL on CaSR and ARP in hippocampus tissue of rats with epilepsy following dementia in Cochrane Library, EMBASE, MEDLINE, CINAHL, AMED, and CNKI from inception to the present. We present search strategy with details for Cochrane Library. We will adapt similar search strategy to other electronic bibliographic databases. Other sources - We will also search other sources to avoid missing potential studies, such as conference proceedings, associated references lists of included studies, and ongoing trials from websites of clinical trial registry.

Main outcome(s): Outcomes include protein and gene expressions of CaSR, cFos, Caspase-3, Bcl-2, Bax, Neural Cell Adhesion Molecule 1, Proliferating cell nuclear antigen, CyclinD1, livin; and levels of NO, NOS, and Interleukin 10 in hippocampus tissue of rats.

Data management: Two authors will independently extract data from eligible study in accordance with a predefined standardized data extraction sheet. If we identify any disagreement, we will invite another author to solve it through discussion. We will extract the following information of title, first author, year of publication, species, gender, study methods, details of intervention and control (e.g. time, dosage, and duration), outcome indicators, results, and findings.

Quality assessment / Risk of bias analysis: The risk of bias of eligible CCSs will be performed by two independent authors using Newcastle-Ottawa Scale. If there is conflict between two authors, we will invite another author to clear up such division.

Strategy of data synthesis: We will use RevMan 5.3 software to analyze and synthesize outcome data. Treatment effect of outcome indicators will be estimated using risk ratio and $95 \%$ confidence intervals (Cls) for dichotomous data, and mean difference (MD) or standardized MD and $95 \%$ Cls for continuous data.We will examine heterogeneity across CCSs using Cochrane $I^{2}$ test. If the value of $I^{2} \leq 50 \%$ is identified, it means reasonable heterogeneity, and we will place a fixedeffects model. Under such situation, we will carry out meta-analysis if sufficient data is collected with sufficient similarity on the same outcome indicator. If the value of $\mathrm{I}^{2}>$ $50 \%$ is found, it indicates substantial heterogeneity, and we will apply a randomeffects model. We will identify any possible sources of significant heterogeneity. If meta-analysis is deemed not to be performed, we will report study results by a narrative description.

Subgroup analysis: We will undertake a subgroup analysis based on the different characteristics of subjects, treatment schedules, and outcome variables.

Sensibility analysis: We will carry out a sensitivity analysis to check the stability and robustness of study results according to the methodological quality, sample size, and missing data.

Country(ies) involved: China. 
Keywords: Epilepsy; dementia; Spore Powder of Ganoderma Lucidum.

Contributions of each author:

Author 1 - Li-hong Qin.

Author 2 - Chen Wang.

Author 3 - Xiao-xue Jiang.

Author 4 - You Song.

Author 5 - Yao Feng.

Author 6 - Li-wei Qin.

Author 7 - Shu-ping Zhang. 